\title{
Quantitative mathematical objective evaluation of contrast-enhanced spectral mammogram in the assessment of response to neoadjuvant chemotherapy and prediction of residual disease in breast cancer
}

Amr Farouk Ibrahim Moustafa ${ }^{1 *}$ D, Rasha Mohammed Kamal², Mohammed Mohammed Mohammed Gomaa', Shaimaa Mostafa ${ }^{3,4}$, Roaa Mubarak ${ }^{4}$ and Mohamed El-Adawy ${ }^{4}$

\begin{abstract}
Background: The aim of the study is to initiate a new quantitative mathematical objective tool for evaluation of response to neoadjuvant chemotherapy (NAC) and prediction of residual disease in breast cancer using contrastenhanced spectral mammography (CESM). Forty-two breast cancer patients scheduled for receiving NAC were included. All patients underwent two CESM examinations: pre and post NAC. To assess the response to neoadjuvant chemotherapy, we used a mathematical image analysis software that can calculate the difference in the intensity of enhancement between the pre and post neoadjuvant contrast images (MATLAB and Simulink) (Release 2013b). The proposed technique used the pre and post neoadjuvant contrast images as inputs. The technique consists of three main steps: (1) preprocessing, (2) extracting the region of interest (ROI), and (3) assessment of the response to chemotherapy by measuring the percentage of change in the intensity of enhancement of malignant lesions in the pre and post neoadjuvant CESM studies using a quantitative mathematical technique. This technique depends on the analysis of number of pixels included within the ROI. We compared this technique with the currently used method of evaluation: RECIST 1.1 (response evaluation criteria in solid tumors 1.1) and using another combined response evaluation approach using both RECIST 1.1 in addition to a subjective visual evaluation. Results were then correlated with the postoperative pathology evaluation using MillerPayne grades. For statistical evaluation, patients were classified into responders and non-responders in all evaluation methods.

(Continued on next page)
\end{abstract}

\footnotetext{
* Correspondence: amrfaroukmoustafa@cu.edu.eg

${ }^{1}$ Department of Diagnostic and Interventional Radiology, National Cancer

Institute, Cairo University, Cairo, Egypt

Full list of author information is available at the end of the article
} 
(Continued from previous page)

Results: According to the Miller-Payne criteria, 39/42 (92.9\%) of the participants were responders (MillerPayne grades III, IV, and IV) and 3/42 (7.1\%) were non-responders (Miller-Payne grades I and II). Using the proposed technique, 39/39 (100\%) were responders in comparison to 38/39 patients (97.4\%) using the combined criteria and 34/39 (87.2\%) using the RECIST 1.1 evaluation. The calculated correlation coefficient of the proposed quantitative objective mathematical technique, RECIST 1.1 criteria, and the combined method was $0.89,0.59$, and 0.69 respectively. With classification of patients into responder and non-responders, the objective mathematical evaluation showed higher sensitivity, positive and negative predictive values, and overall accuracy $(100 \%, 97.5 \%, 100 \%$, and $85.7 \%$ respectively) compared to RECIST 1.1 evaluation (87.2\%, 97.1\%, $28.6 \%$, and $54.8 \%$ respectively) and the combined response method $(97.4 \%, 97.4 \%, 66.7 \%$, and $85.7 \%$ respectively).

Conclusion: Quantitative mathematical objective evaluation using CESM images allows objective quantitative and accurate evaluation of the response of breast cancer to chemotherapy and is recommended as an alternative to the subjective techniques as a part of the pre-operative workup.

Keywords: Contrast-enhanced spectral mammogram, Neoadjuvant chemotherapy, Breast cancer

\section{Background}

Pre-operative neoadjuvant chemotherapy (NAC) is being continuously more employed in the control of locally advanced breast cancers and even in lower tumor stages enabling breast-conserving surgery in patients that would otherwise undergo mastectomy [1-3]. The use of NAC also enables physicians to assess tumor response in vivo. Pathological complete response after NAC may be considered an independent good prognostic factor. In fact, a pathological complete response has been associated with significantly improved disease-free survival and overall survival rates $[1,4]$.

A modality that enables the assessment of tumor response and accurately detects any residual disease has been always pursued [1]. Conventional methods, including clinical examination, ultrasonography (US), and mammography, have been proved to be of limited efficacy. MRI has been always looked at as the modality of choice in evaluating response to NAC as it allows assessment of both change in the tumor size and change in its morphology characteristics [4-6]. In addition to these MRI merits, studies assessing the response to neoadjuvant chemotherapy using semi-quantitative and quantitative MRI techniques allow early prediction of response even after one or two cycles. Identifying nonresponders early allows amending treatment plans and facilitates the setting of tailored treatment regimens for each specific breast cancer patient [7-12].

The aim of this study is to initiate a new quantitative mathematical objective tool for evaluating the response of malignant breast mass lesions to neoadjuvant chemotherapy and allows accurate assessment of residual disease using contrast-enhanced spectral mammography (CESM) in comparison with response evaluation criteria in solid tumors 1.1 (RECIST 1.1) and a combined evaluation method (quantitative and qualitative). To our knowledge, this work is the first research that uses objective and quantitative mathematical evaluation using CESM. Previous published articles in literature in the same setting have only used subjective qualitative assessment methods and quantitative assessments only relied on measuring change in tumor size based on RECIST and measuring size of residual disease which is liable to over or under estimation $[2,13]$. None of these researches discussed quantitative change in intensity of contrast uptake which reflects actual change in tumor cell activity.

\section{Methods \\ Patients}

Forty-two patients with pathologically proved breast cancer based on the tumor tissues obtained by core needle biopsy were enrolled in this study. They were all scheduled to receive NAC according to the decision of the multidisciplinary breast cancer tumor board. All patients underwent two separate CESM examinations; pre and post neoadjuvant chemotherapy.

The maximum interval between the post-NAC study and surgery was 10 days.

The study protocol was approved by the Institutional Review Board and informed written consent was applied for the used data of the enrolled individuals.

Patients who were not candidates for NAC, patients with distant metastases, pregnant females, those with a history of allergy, or renal impairment were excluded from the study.

Examinations were performed using the GE Senographe Essential mammography unit. 


\section{Assessment of response to neoadjuvant chemotherapy using CESM}

\section{A. RECIST 1.1 evaluation}

- Quantitative assessment was performed by measuring the longest dimension of the target lesions (two lesions per organ) before and after NAC. After interpreting the difference in size between both measurements, response to NAC was then classified according to the response evaluation criteria in solid tumors (RECIST 1.1) [14, 15]. For statistical analysis, lesions showing stable or progressive response were classified as nonresponders while lesions showing partial or complete response were classified as responders.

B. Combined quantitative and qualitative assessment

- The combined assessment was previously proposed by the involved researchers [16]. It depends on a combination of measuring the largest diameter of the target lesion together with subjective identification of the difference in intensity of contrast uptake before and after NAC. For statistical analysis, patients showing progressive, stable, or poor response were classified as non-responders while patients showing moderate, marked, or complete response were classified as responders (Table 1).

C. Quantitative mathematical objective evaluation

- A new objective mathematical tool for evaluation of response to neoadjuvant chemotherapy and assessment of residual disease was introduced depending on a combination of the summation of the number of pixels and their intensity within the area of interest before and after NAC. A mathematical image analysis software (MATLAB and Simulink) (Release 2013b) is used in the following steps:

Pre-processing: Applying the step of preprocessing plays a vital role to remove artifacts, labels, and increase the quality of the image [17]. The pre-processing of the images is prepared to reduce the computational rate and exploit the probability of accuracy [17]. It also involves denoising and improving the contrast of the images by removing artifacts. This is followed by resizing all the images to a fixed size [17-20]. Input images were converted to grayscale intensity image. The main target of conversion to grayscale is to eliminate the hue and saturation information while retaining the luminance.

1. Image segmentation: The breast contour is first separated from the image back ground. Then a draggable rectangular is generated manually to select the area which involves the malignant mass (region of interest) both in the pre and post NAC images. Automatic thresholding was then applied to extract the malignant mass in the images before and after taking chemotherapy as shown in Fig. 1.

2. Image thresholding is an effective method of splitting an image into a foreground and background and also is the most effective in images with high levels of contrast. This division into parts is often based on the characteristics of the pixels in the image. Automatic thresholding was done by Singh AK and Gupta B.2015 [21] that was used to separate pixels of malignant mass from the normal region. A white patch covering the malignant mass was obtained.

3. Finding the ratio of response: Executing mathematical operations are performed to deduce the response of cancerous lesions to NAC. After applying thresholding, the number of pixels within the region of interest (ROI) is summed up both in the pre and the post NAC images to

Table 1 The combined evaluation response approach

\begin{tabular}{lll}
\hline Type & Tumor largest diameter & Intensity of enhancement \\
\hline - Progressive disease & Increase & $+/-$ change in the intensity \\
- Stable disease & No change in size & No change \\
- Poor response & Up to 30\% decrease & Decrease in intensity \\
- Moderate response & $30-60 \%$ decrease & Decrease in intensity \\
- Marked response & $>60 \%$ decrease & Residual faint enhancement \\
- Complete Response & No residual lesion seen & \\
\hline
\end{tabular}


(a)

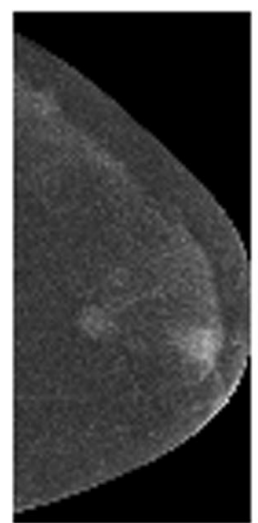

(d)

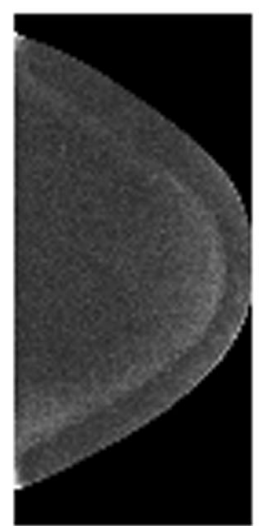

(b)
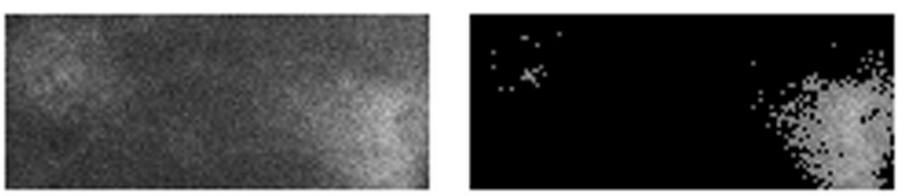

(e)
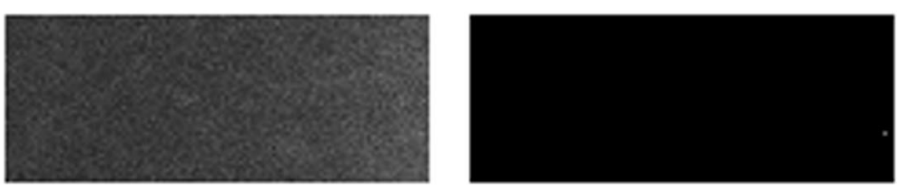

(c)

(i)

Fig. 1 a, $\mathbf{d}$ The original image. $\mathbf{b}$, e The suspicious region and $\mathbf{c}, \mathbf{f}$ after applying thresholding

identify the intensity of enhancement. The size of each region can be determined by counting the enclosed pixels according to the following equation:

$A=\Sigma \mathrm{Pi}$ (where: $\mathrm{Pi}$ is the intensity value of pixels)
Then the response to NAC is measured by calculating the ratio between the areas in the pre and post NAC images to according to the following equation:

$$
\begin{aligned}
& \sum_{i=0}^{n} P i \text { After chemo } \\
& R=\overline{\sum_{i=0}^{n} P i \text { Before chemo }}
\end{aligned}
$$

Table 2 Miller-Payne grading system

\begin{tabular}{ll}
\hline Grade & Histopathology findings \\
\hline Grade 1 & $\begin{array}{l}\text { No change or some alteration to individual malignant } \\
\text { cells, but no reduction in overall cellularity }\end{array}$ \\
Grade 2 & $\begin{array}{l}\text { Minor loss of tumor cells, but overall cellularity still } \\
\text { high; up to 30\% }\end{array}$ \\
Grade 3 & $\begin{array}{l}\text { Between an estimated 30-90\% reduction in tumor cells } \\
\text { Grade 4 }\end{array}$ \\
& $\begin{array}{l}\text { Marked disappearance of tumor cells such that only } \\
\text { small clusters or widely dispersed individual cells remain; } \\
\text { more than 90\% loss of tumor cells }\end{array}$ \\
Grade 5 & $\begin{array}{l}\text { No malignant cells identifiable in sections from the site } \\
\text { of the tumor; only vascular fibro-elastic stroma remains } \\
\text { often containing macrophages. However, DCIS may be } \\
\text { present }\end{array}$ \\
\hline
\end{tabular}

Table 3 Histopathological and molecular subtypes of the patients enrolled in the study

\begin{tabular}{ll}
\hline Histological Subtype: & Number and \\
• Invasive ductal carcinomas (IDC). & percentage \\
- Invasive lobular carcinomas (ILC). & $\cdot 36 / 42$ tumors (85.7\%) \\
• Mixed invasive ductal and lobular & $\cdot 4 / 42$ tumors $(9.5 \%)$ \\
carcinomas. & $\cdot 1 / 42$ tumor $(2.4 \%)$ \\
• Invasive tubular carcinoma (ITC). & $\cdot 1 / 42$ tumor $(2.4 \%)$ \\
Biomarker s status of the tumors: & Number and \\
HER2 over-enriched cancers. & percentage \\
HER2-negative/HR-positive cancer & $9 / 42$ were $(21.4 \%)$ \\
Triple-negative cancers. & $17 / 42(40.45 \%)$ \\
Luminal A cancer. & $12 / 42(28.6 \%)$ \\
& $4 / 42(9.5 \%)$ \\
\hline
\end{tabular}




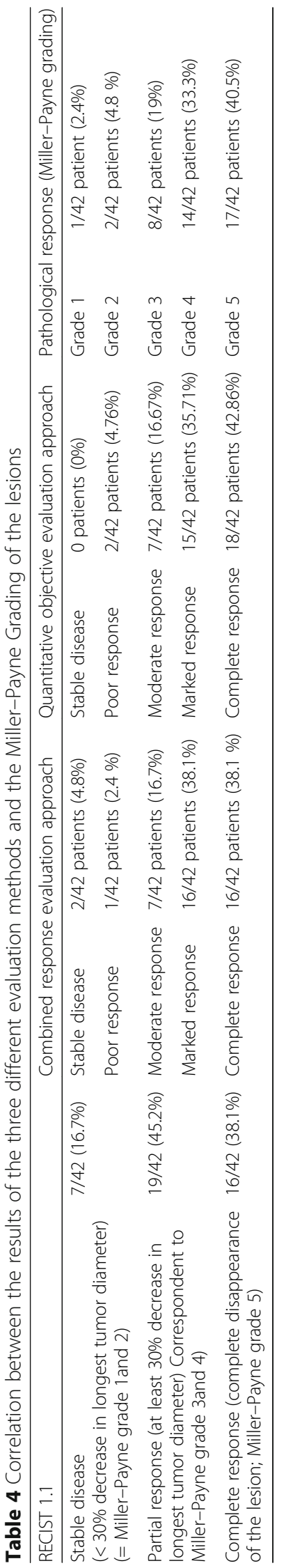


Finally, we converted this ratio to fit with the correspondent pathology based Miller-Payne grades (Table 2).

\section{Histopathology}

Tumor regression was quantitatively graded by two independent pathologists in the surgical biopsy specimens based on the Miller-Payne grading system by identifying residual tumor cellularity NAC [22] (Table 2).

Patients were divided into two groups: pathologic responders (lesions showing Miller-Payne grades 3, 4, and 5), and pathologic non-responders (lesions showing Miller-Payne grades 1 and 2).

\section{Statistical analysis}

Data was coded and entered using the statistical package SPSS (Statistical Package for the Social Sciences) version 24. Standard diagnostic indices including sensitivity, specificity, positive and negative predictive values (PPV and NPV), and likelihood ratios were calculated. For comparing categorical data, Chi-square $\left(x^{2}\right)$ test was performed. The exact test was used instead when the expected frequency is less than 5. Correlations between quantitative variables were done using Pearson correlation coefficient. A $P$ value less than 0.05 was considered statistically significant.

\section{Results}

The study included 42 patients with pathologically proved breast cancer. The histopathological and molecular subtypes of the 42 malignant lesions are demonstrated in Table 3.

The correlation between the results of the three different evaluation methods and the Miller-Payne grading of the lesions is demonstrated in Table 4 and Figs. 2, 3, and 4 .

The highest correlation coefficient was between the values obtained from the quantitative objective evaluation method ( $r: 0.8944)$ as demonstrated in Table 5.

Patients were then classified as responders and nonresponders as shown in Table 6 to facilitate the calculation of the diagnostic indices of the three evaluation methods. Response was best evaluated using the quantitative objective evaluation were 39/39 lesions matched the Miller-Payne evaluation. There was only one false positive responder by the three evaluation methods.

The last step was to calculate the diagnostic indices and overall accuracy of the three evaluation methods as demonstrated in Table 7. The objective evaluation method again scored the highest indices (Fig. 5).

\section{Discussion}

Neoadjuvant chemotherapy permits in vivo testing of response and thus allows monitoring of individual tumor



Fig. 2 The correlation between the combined response evaluation and the corresponding Miller-Payne grading 


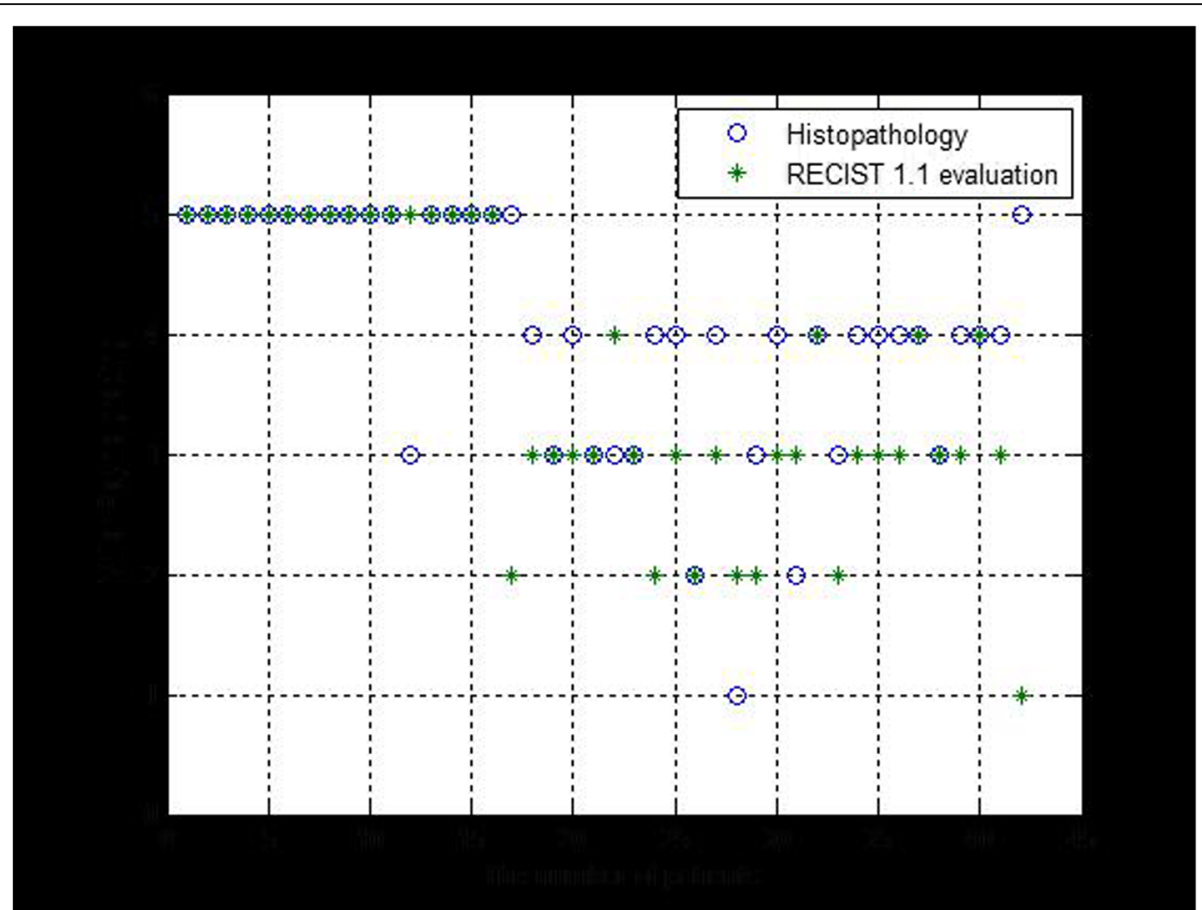

Fig. 3 The correlation between RECIST1.1 evaluation and the corresponding Miller-Payne grading

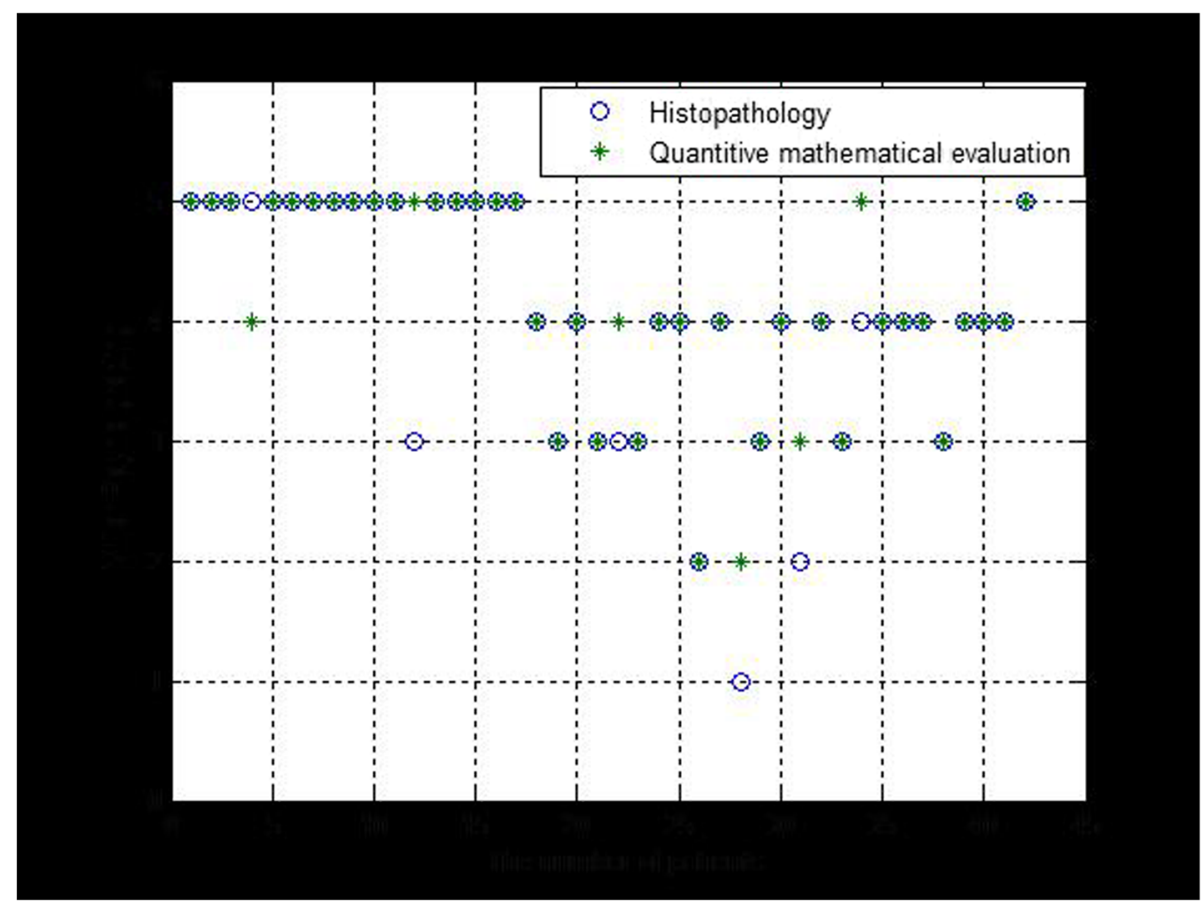

Fig. 4 The correlation between the quantitative mathematical evaluation and the corresponding Miller-Payne grading 
Table 5 Correlation coefficient between the different evaluation methods and the Miller-Payne Grade of the malignant lesions

\begin{tabular}{lll}
\hline & $\begin{array}{l}\text { Correlation } \\
\text { coefficient }\end{array}$ & Accuracy \\
\hline Combined response evaluation & 0.6899 & $85.7 \%$ \\
RECIST 1.1 Evaluation & 0.5950 & $54.8 \%$ \\
Quantitative mathematical objective evaluation & 0.8944 & $85.7 \%$ \\
\hline
\end{tabular}

response. Down staging malignant lesions allows reduction in surgical complications, more conservative surgeries, and safer axillary dissections [23, 24].

The utilization of noninvasive imaging in monitoring the response of malignant breast lesions to NAC has become crucial. It may help distinguish patients who are expected to achieve a pathologic complete response from those who show no appreciable response early in the treatment course. Identifying non-responders early enough allows the planning of alternative treatment options and avoids unnecessary toxicity [7]. Many modalities have been suggested to evaluate tumor response to NAC but all of which were accused of having an unpretentious accuracy [1]. Dynamic contrast MRI has long been considered the best imaging modality for both monitoring tumor response to NAC and for the assessment of residual disease extent without competition.

CESM has emerged as one of the most promising imaging modalities with comparable sensitivity and specificity to MRI. In the neoadjuvant setting, a few studies compared the utility of CESM and MRI. In the study performed by Lotti et al., the size of residual disease after NAC was measured by both modalities based on the RECIST 1.1 criteria. They reported good correlation between both modalities (agreement: 0.76) and thus they concluded that CESM is as reliable as MRI and may be used as an adequate alternative [2]. In another study, Patel et al. concluded that the accuracy of CESM was equivalent to MRI in assessing residual disease after NAC [13]. However, these two studies depend on residual tumor size assessment. None of these studies discussed change in tumor functions which was previously investigated by quantitative MRI techniques. Another major disadvantage of the methods used in these studies is that to identify changes in tumor sizes, neoadjuvant therapy first induces cell changes that end by cell death which is then followed by size changes. In a systematic review by Lobbes et al., they stated that there is tendency of over and under estimation of response depending on tumor size alone which may both affect cosmetic outcomes and amount free margin status after operative intervention $[25,26]$.

To overcome these disadvantages, quantitative measures have also been tested and validated as reliable early predictors of response [8]. In the current study, we assessed a new quantitative objective tool in comparison to the previously used RECIST 1.1 criteria and a proposed combined quantitative and subjective qualitative approach in assessing the response to NAC using CESM.

The current cohort study included 42 patients who were diagnosed with breast cancer and were scheduled to receive neoadjuvant chemotherapy. Patients underwent two CESM studies (pre and post NAC) and were followed up along their treatment course. According to histopathology revision of core and surgical biopsy specimens, the commonest histopathology subtype was invasive ductal carcinomas (IDC) grades 2 and $3(36 / 42,85.7 \%)$ and the commonest molecular subtype was hormone receptor positive tumors (17/ $42,40.45 \%)$ followed by triple-negative tumors (12/42, $28.6 \%)$. Complete pathological response was achieved in $17 / 42(40.45 \%)$ lesions; ten triple-negative tumors and seven Her2-positive lesions. This coincides with what is stated in literature that compared with hormone receptor-positive tumors, HER2-overexpression, and triple-negative subtypes are more sensitive to NAC [23, 27] (Fig. 6).

We started by classifying the response; using the three assessment modalities, into grades that parallel the

Table 6 Comparison between the numbers of patients classified as responders/non-responders using each of the three evaluations versus pathology base Miller-Payne grading

\begin{tabular}{|c|c|c|c|c|c|}
\hline & & \multicolumn{4}{|c|}{ Pathologic Response } \\
\hline & & \multicolumn{2}{|l|}{ Responder } & \multicolumn{2}{|l|}{ Non-responder } \\
\hline & & Count $(n=39)$ & $\%$ & Count $(n=3)$ & $\%$ \\
\hline \multirow[t]{2}{*}{ Quantitative objective evaluation } & Responder $(n=40)$ & 39 & $100 \%$ & 1 & $33.3 \%$ \\
\hline & Non-responder $(n=2)$ & 0 & $0 \%$ & 2 & $66.7 \%$ \\
\hline \multirow[t]{2}{*}{ RECIST 1.1 Evaluation } & Responder $(n=35)$ & 34 & $87.2 \%$ & 1 & $33.3 \%$ \\
\hline & Non-responder $(n=7)$ & 5 & $12.8 \%$ & 2 & $66.7 \%$ \\
\hline \multirow[t]{2}{*}{ Combined response evaluation } & Responder $(n=39)$ & 38 & $97.4 \%$ & 1 & $33.3 \%$ \\
\hline & Non-responder $(n=3)$ & 1 & $2.6 \%$ & 2 & $66.7 \%$ \\
\hline
\end{tabular}




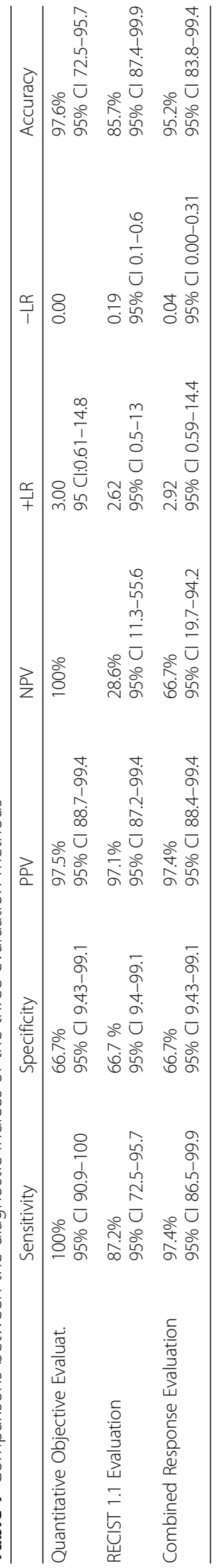




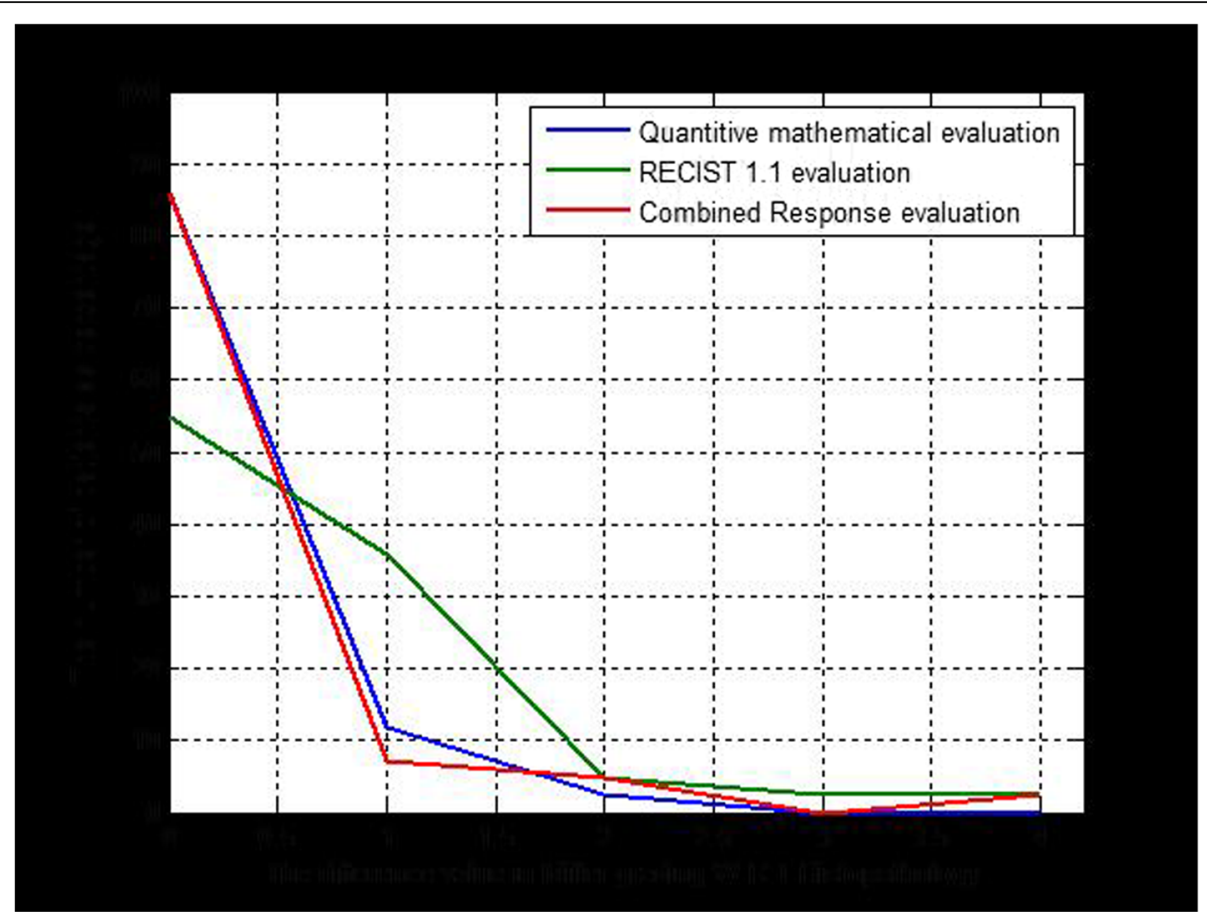

Fig. 5 Comparison between the quantitative mathematical objective evaluation, RECIST 1.1 method, and the combined method in correlation to the corresponding histopathology Miller-Payne grading

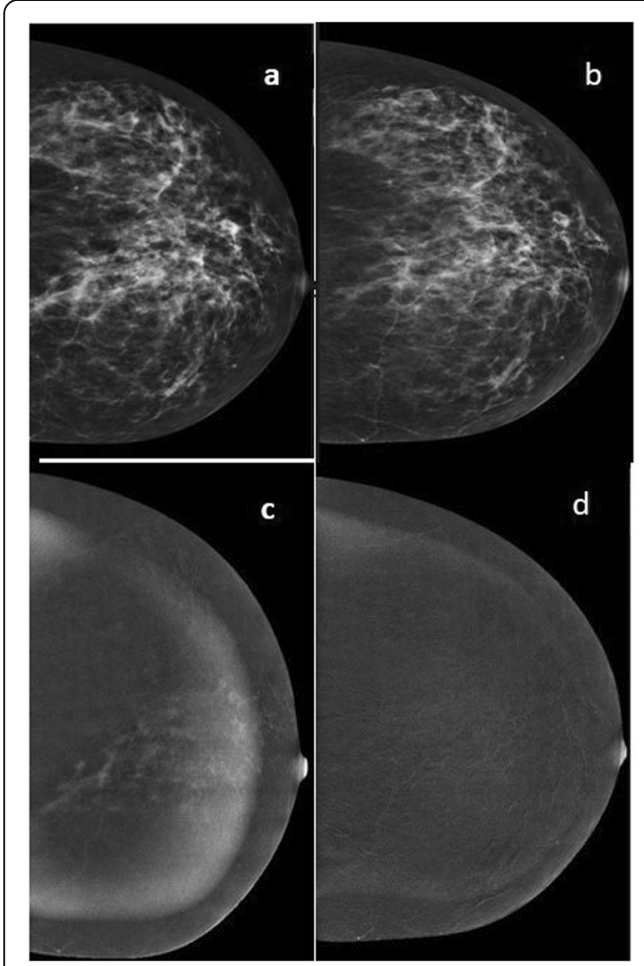

Tumor Region befor chemo



e


Tumor Region after chemo


Fig. 6 A 56-year-old patient with ILC of the left breast received neoadjuvant chemotherapy. a Pre NAC mammogram CC view showing a left breast focal asymmetry. b Post NAC mammogram CC view showing reduction in size. $\mathbf{c}$ Pre NAC CESM CC view grouped enhancing lesions. $\mathbf{d}$ Post NAC CESM CC view showing no residual pathological enhancement. e Quantitative mathematical objective evaluation. RECIST 1.1 classified the patient as partial responder. Combined response evaluation classified this patient as a complete responder. Quantitative mathematical objective evaluation showed $99 \%$ regression. Pathological evaluation confirmed this patient as acomplete responder (Miller-Payne grade 5) 
Miller Payne Grades. The quantitative objective evaluation scored the highest correlation ( $r: 0.89)$ with the corresponding Miller-Payne Grade. This was followed by the combined evaluation $(r: 0.68)$ and the lowest scored value was for the RECIST 1.1 criteria $(r: 059)$. In the study performed by Iotti et al., CESM and MRI measurements were highly correlated [2]. They even found that CESM can better predict complete pathological response better than MRI (Lin's coefficient: 0.81 for CESM and 0.59 for MRI). In another study, performed by Barra et al., they also proved that CESM measurements showed a strong, steady correlation with the pathology residual tumor size $(R: 0.76)$, a value which is slightly higher than that scored by the RECIST 1.1 criteria in the current study [28] (Fig. 7).

To facilitate the calculation of the diagnostic indices of the three assessment methods, patients had to be re-grouped into either responders or non-responders. Using the three methods, only one case was considered a false positive responder. Errors in contrast administration or timing of the imaging could not be excluded. No false negative cases were reported by the quantitative objective evaluation as compared to 1 case by the combined evaluation and 5 cases for the RECIS1.1 evaluation.

The reported sensitivity, specificity, and accuracy of DCE-MRI for residual disease evaluation are 86-92\%, 60$89 \%$, and $76-90 \%$, respectively [29-35]. The quantitative mathematical objective evaluation showed higher sensitivity, positive and negative predictive values, as well as overall accuracy compared to the evaluation based on RECIST 1.1 alone and combined response evaluation. (100\%, 97.5\%, $100 \%$, and $97.6 \%$ respectively compared to $87.2 \%, 97.1 \%$, $28.6 \%$, and $85.7 \%$ for RECIST 1.1 and $97.4 \%, 97.4 \%, 66.7 \%$, and $95.2 \%$ for combined response evaluation). Previous studies reported nearly similar indices, although they were mainly based on residual tumor size assessment. Barra et al. [28] reported no false positive cases and the calculated sensitivity, specificity, PPV, and NPV was $83.33 \%, 100 \%, 100 \%$, and $66 \%$ respectively. On the other hand, Iotti et al. [2] reported no false negative cases with a calculated sensitivity and specificity of $100 \%$ and $84 \%$, respectively.

\section{Conclusion}

CESM can be readily used to assess tumor response to NAC with the mathematical objective evaluation and




combined response evaluation providing efficient tools for evaluation of response and residual tumor. They not only allowed size discrepancy assessment but provided information about the functional changes in the residual tumor. The extra-merit of mathematical objective evaluation is avoiding bias in the evaluation.

\section{Abbreviations}

CESM: Contrast-enhanced spectral mammogram; IDC: Invasive ductal carcinomas; ILC: Invasive lobular carcinomas; ITC: Invasive tubular carcinoma; NAC: Neoadjuvant chemotherapy; RECIST 1.1: Response evaluation criteria in solid tumors 1.1; ROI: Region of interest

\section{Acknowledgments}

This research was carried out at Baheya Charity Women's Cancer Hospital which is fully equipped by modern machines for breast cancer diagnosis. We want to thanks our colleagues who helped us to do such research work.

\section{Availability of data and materials}

The datasets used and analyzed during the current study are available from the corresponding author on reasonable request.

\section{Authors' contributions}

AM wrote the manuscript and responsible for correspondence to journal. SM collected patient data and participated in its design. MG image processing and collection of patient's images. RM and ME participated in the design of the study and performed the statistical analysis. RK conceived of the study, and participated in its design and coordination and helped to draft the manuscript. All authors read and approved the final manuscript.

\section{Funding}

No funding sources.

\section{Competing interest}

The authors declare that they have no competing interests .

\section{Ethics approval and consent to participate}

The study was approved by the ethical committee of "Baheya Foundation for Early Detection \& Treatment of Breast Cancer" with ethical committee approval number R-17-10-28 and approval date 12/2017. An informed written consent was taken from all subjects.

\section{Consent for publication}

All patients included in this research gave written informed consent to publish the data contained within this study.

\section{Author details \\ 'Department of Diagnostic and Interventional Radiology, National Cancer Institute, Cairo University, Cairo, Egypt. ${ }^{2}$ Department of Diagnostic and Interventional Radiology, Faculty of Medicine, Cairo University, Cairo, Egypt. ${ }^{3}$ Communication \& Electronics Engineering Department, Institute of Engineering, Canadian International College (CIC), Giza, Egypt. ${ }^{4}$ Electronics, Communication, and Computer Engineering Department, Faculty of Engineering, Helwan University, Cairo, Egypt.}

Received: 5 July 2019 Accepted: 6 September 2019

Published online: 22 October 2019

\section{References}

1. Zhou J, Li G, Sheng F, Qiao P, Zhang H, Xing X (2016) Magnetic resonance imaging evaluation of residual tumors in breast cancer after neoadjuvant chemotherapy: surgical implications. Acta radiol 57:529-537. https://doi.org/ $10.1177 / 0284185115597263$

2. Iotti V, Ravaioli S, Vacondio R, Coriani C, Caffarri S, Sghedoni R, Nitrosi A, Ragazzi M, Gasparini E, Masini C, Bisagni G, Falco G, Ferrari G, Braglia L, Del Prato A, Malavolti I, Ginocchi V, Pattacini P (2017) Contrast-enhanced spectral mammography in neoadjuvant chemotherapy monitoring: a comparison with breast magnetic resonance imaging. Breast Cancer Res 19: 106. https://doi.org/10.1186/s13058-017-0899-1
3. Mamounas EP (2015) Impact of neoadjuvant chemotherapy on locoregional surgical treatment of breast cancer. Ann Surg Oncol 22:1425-1433. https:// doi.org/10.1245/s10434-015-4406-6

4. El bakoury EAEM, Khalil RF, Abdelhamid AEM, Darwish AMA, Hassan HHM (2017) Diffusion weighted imaging in early prediction of neoadjuvant chemotherapy response in breast cancer. Egypt J Radiol Nucl Med 48:529535. https://doi.org/10.1016/J.EJRNM.2017.03.021

5. Lee J, Kim SH, Kang BJ (2018) Pretreatment prediction of pathologic complete response to neoadjuvant chemotherapy in breast cancer: perfusion metrics of dynamic contrast enhanced MRI. Sci Rep 8:9490. https://doi.org/10.1038/s41598-018-27764-9

6. Goorts B, Dreuning KMA, Houwers JB, Kooreman LFS, Boerma E-JG, Mann RM, Lobbes MBI, Smidt ML (2018) MRI-based response patterns during neoadjuvant chemotherapy can predict pathological (complete) response in patients with breast cancer. Breast Cancer Res 20:34. https://doi.org/10.1186/ s13058-018-0950-x

7. Rauch GM, Adrada BE, Kuerer HM, van la Parra RFD, Leung JWT, Yang WT (2017) Multimodality imaging for evaluating response to neoadjuvant chemotherapy in breast cancer. Am J Roentgenol 208:290-299. https://doi. org/10.2214/AJR.16.17223

8. Tudorica A, Oh KY, Chui SY-C, Roy N, Troxell ML, Naik A, Kemmer KA, Chen Y, Holtorf ML, Afzal A, Springer CS, Li X, Huang W, Huang W (2016) Early prediction and evaluation of breast cancer response to neoadjuvant chemotherapy using quantitative DCE-MRI. Transl Oncol 9:8-17. https://doi. org/10.1016/j.tranon.2015.11.016

9. Pickles MD, Lowry M, Manton DJ, Turnbull LW (2015) Prognostic value of DCE-MRI in breast cancer patients undergoing neoadjuvant chemotherapy: a comparison with traditional survival indicators. Eur Radiol 25:1097-1106. https://doi.org/10.1007/s00330-014-3502-5

10. Woolf DK, Padhani AR, Taylor NJ, Gogbashian A, Li SP, Beresford MJ, Ah-See M-L, Stirling J, Collins DJ, Makris A (2014) Assessing response in breast cancer with dynamic contrast-enhanced magnetic resonance imaging: Are signal intensity-time curves adequate? Breast Cancer Res Treat 147:335-343. https://doi.org/10.1007/s10549-014-3072-x

11. Li $X$, Kang H, Arlinghaus LR, Abramson RG, Chakravarthy AB, Abramson VG, Farley J, Sanders M, Yankeelov TE (2014) Analyzing spatial heterogeneity in DCE- and DW-MRI parametric maps to optimize prediction of pathologic response to neoadjuvant chemotherapy in breast cancer. Transl Oncol 7:1422. https://doi.org/10.1593/tlo.13748

12. Huang W, Li X, Chen Y, Li X, Chang M-C, Oborski MJ, Malyarenko DI, Muzi M, Jajamovich GH, Fedorov A, Tudorica A, Gupta SN, Laymon CM, Marro KI, Dyvorne HA, Miller JV, Barbodiak DP, Chenevert TL, Yankeelov TE, Mountz JM, Kinahan PE, Kikinis R, Taouli B, Fennessy F, Kalpathy-Cramer J (2014) Variations of dynamic contrast-enhanced magnetic resonance imaging in evaluation of breast cancer therapy response: a multicenter data analysis challenge. Transl Oncol 7:153-166. https://doi.org/10.1593/tlo.13838

13. Patel BK, Hilal T, Covington M, Zhang N, Kosiorek HE, Lobbes M, Northfelt DW, Pockaj BA (2018) Contrast-enhanced spectral mammography is comparable to mri in the assessment of residual breast cancer following neoadjuvant systemic therapy. Ann Surg Oncol 25:1350-1356. https://doi. org/10.1245/s10434-018-6413-x

14. Tozaki M, Kobayashi T, Uno S, Aiba K, Takeyama H, Shioya H, Tabei I, Torium Y, Suzuki M, Fukuda K (2006) Breast-conserving surgery after chemotherapy: value of MDCT for determining tumor distribution and shrinkage pattern. Am J Roentgenol 186:431-439. https://doi.org/10.2214/AJR.04.1520

15. Schwartz LH, Litière S, de Vries E, Ford R, Gwyther S, Mandrekar S, Shankar L, Bogaerts J, Chen A, Dancey J, Hayes W, Hodi FS, Hoekstra OS, Huang EP, Lin N, Liu Y, Therasse P, Wolchok JD, Seymour L (2016) RECIST 1.1-Update and clarification: From the RECIST committee. Eur J Cancer 62:132-137. https:// doi.org/10.1016/j.ejca.2016.03.081

16. Abdelhameed S, Kamal RM, Moustafa Al, Gomaa MM, Omniya M (2019) No Title. Eur Congr Radiol Voice EPOS stage 2:208 C2812-Oncol Imaging

17. Mostafa S, Mubarak R, El-Adawy M (2018) Breast cancer detection using polynomial fitting for intensity spreading within ROls, pp 129-139

18. Rehman AU, Chouhan N, Khan A (2015) Diverse and discrimintative features based breast cancer detection using digital mammography. 2015 13th International Conference on Frontiers of Information Technology (FIT). IEEE: 234-239

19. Appukuttan A, L. S (2015) Breast cancer-early detection and classification techniques: a survey. Int J Comput Appl 132:9-13 . doi: https://doi.org/10. 5120/ijca2015907557 
20. Sahar M, Nugroho HA, Tianur Al, Choridah L (2016) Automated detection of breast cancer lesions using adaptive thresholding and morphological operation. In: 2016 International Conference on Information Technology Systems and Innovation (ICITSI). IEEE, pp 1-4

21. Singh AK, Gupta B (2015) A novel approach for breast cancer detection and segmentation in a mammogram. Procedia Comput Sci 54:676-682. https:// doi.org/10.1016/j.procs.2015.06.079

22. Ogston KN, Miller ID, Payne S, Hutcheon AW, Sarkar TK, Smith I, Schofield A, Heys SD (2003) A new histological grading system to assess response of breast cancers to primary chemotherapy: prognostic significance and survival. Breast 12:320-327

23. Hartkopf AD, Müller V, Wöckel A, Lux MP, Janni W, Nabieva N, Taran F-A, Ettl J, Lüftner D, Belleville E, Schütz F, Fasching PA, Fehm TN, Kolberg H-C, Overkamp F, Schneeweiss A, Tesch H (2019) Update breast cancer 2019 part 1 -implementation of study results of novel study designs in clinical practice in patients with early breast cancer. Geburtshilfe Frauenheilkd 79: 256-267. https://doi.org/10.1055/a-0842-6614

24. Fernandez-Gonzalez S, Falo C, Pla MJ, Pernas S, Bajen M, Soler T, Ortega R, Quetglas C, Perez-Martin X, Fernandez Montoli ME, Campos M, VarelaRodriguez M, Ponce J, Garcia-Tejedor A (2018) The shift from sentinel lymph node biopsy performed either before or after neoadjuvant Systemic therapy in the clinical negative nodes of breast cancer patients. Results, and the advantages and disadvantages of both procedures. Clin Breast Cancer 18: 71-77. https://doi.org/10.1016/j.clbc.2017.08.014

25. Lobbes MBI, Prevos R, Smidt M, Tjan-Heijnen VCG, van Goethem M, Schipper R, Beets-Tan RG, Wildberger JE (2013) The role of magnetic resonance imaging in assessing residual disease and pathologic complete response in breast cancer patients receiving neoadjuvant chemotherapy: a systematic review. Insights Imaging 4:163-175. https://doi.org/10.1007/ s13244-013-0219-y

26. Fowler AM, Mankoff DA, Joe BN (2017) Imaging neoadjuvant therapy response in breast cancer. Radiology 285:358-375. https://doi.org/10.1148/ radiol.2017170180

27. Fasching PA, Gaß P, Hein A (2016) Neoadjuvant treatment of breast cancer-advances and limitations. Breast Care (Basel) 11:313-314. https:// doi.org/10.1159/000452463

28. Barra FR, de Souza FF, Camelo REFA, Ribeiro AC de O, Farage L (2017) Accuracy of contrast-enhanced spectral mammography for estimating residual tumor size after neoadjuvant chemotherapy in patients with breast cancer: a feasibility study. Radiol Bras 50:224-230. https://doi.org/10.1590/ 0100-3984.2016-0029

29. Zahraa Al-Hilli Z, Boughey JC (2016) The timing of breast and axillary surgery after neoadjuvant chemotherapy for breast cancer. Chinese Clin Oncol 5:37-37. https://doi.org/10.21037/cco.2016.03.26

30. Dialani V, Chadashvili T, Slanetz PJ (2015) Role of imaging in neoadjuvant therapy for breast cancer. Ann Surg Oncol 22:1416-1424. https://doi.org/10. 1245/s10434-015-4403-9

31. Bouzón A, Acea B, Soler R, Iglesias Á, Santiago P, Mosquera J, Calvo L, Seoane-Pillado T, García A (2016) Diagnostic accuracy of MRI to evaluate tumour response and residual tumour size after neoadjuvant chemotherapy in breast cancer patients. Radiol Oncol 50:73-79. https://doi.org/10.1515/ raon-2016-0007

32. Yuan Y, Chen X-S, Liu S-Y, Shen K-W (2010) Accuracy of MRI in prediction of pathologic complete remission in breast cancer after Preoperative therapy: a meta-analysis. Am J Roentgenol 195:260-268. https://doi.org/10.2214/AJR. 09.3908

33. Marinovich ML, Houssami N, Macaskill P, Sardanelli F, Irwig L, Mamounas EP, von Minckwitz G, Brennan ME, Ciatto S (2013) Meta-analysis of magnetic resonance imaging in detecting residual breast cancer after neoadjuvant therapy. JNCI J Natl Cancer Inst 105:321-333. https://doi.org/10.1093/jnci/ djs528

34. Marinovich ML, Macaskill P, Irwig L, Sardanelli F, von Minckwitz G, Mamounas E, Brennan M, Ciatto S, Houssami N (2013) Meta-analysis of agreement between MRI and pathologic breast tumour size after neoadjuvant chemotherapy. Br J Cancer 109:1528-1536. https://doi.org/10 1038/bjc.2013.473

35. Marinovich ML, Macaskill P, Irwig L, Sardanelli F, Mamounas E, von Minckwitz G, Guarneri V, Partridge SC, Wright FC, Choi JH, Bhattacharyya M, Martincich L, Yeh E, Londero V, Houssami N (2015) Agreement between MR and pathologic breast tumor size after neoadjuvant chemotherapy, and comparison with alternative tests: individual patient data meta-analysis. BMC Cancer 15:662. https://doi.org/10.1186/s12885-015-1664-4

\section{Publisher's Note}

Springer Nature remains neutral with regard to jurisdictional claims in published maps and institutional affiliations.

\section{Submit your manuscript to a SpringerOpen ${ }^{\circ}$ journal and benefit from:}

- Convenient online submission

- Rigorous peer review

- Open access: articles freely available online

High visibility within the field

- Retaining the copyright to your article

Submit your next manuscript at $\boldsymbol{\nabla}$ springeropen.com 